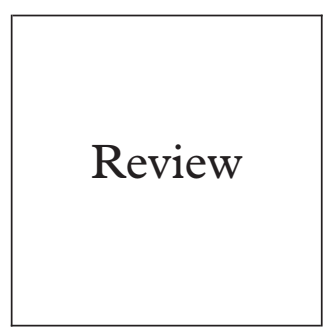

\title{
Syphilis in pregnancy
}

\author{
Mehmet Genç, William J Ledger
}

Syphilis can seriously complicate pregnancy and result in spontaneous abortion, stillbirth, nonimmune hydrops, intrauterine growth restriction, and perinatal death, as well as serious sequelae in liveborn infected children. While appropriate treatment of pregnant women often prevents such complications, the major deterrent has been inability to identify the infected women and get them to undergo treatment. Screening in the first trimester with non-treponemal tests such as rapid plasma reagin (RPR) or venereal disease research laboratory (VDRL) test combined with confirmation of reactive individuals with treponemal tests such as the fluorescent treponemal antibody absorption (FTA-ABS) assay is a cost effective strategy. Those at risk should be retested in the third trimester. Treatment during pregnancy should be with penicillin. In determining a penicillin regimen, the clinician must consider the stage of the maternal infection and the HIV status of the mother. Patients who are allergic to penicillin should be desensitised before treatment. Despite appropriate treatment, as many as $14 \%$ will have a fetal death or deliver infected infants. Treatment may further be complicated by the Jarich-Herxheimer reaction, a complex allergic response to antigens released from dead micro-organisms, which can cause fetal distress and uterine contractions. Thanks to effective intervention strategies and inexpensive penicillin, syphilis rarely complicates pregnancy in the Western world today. In parts of the world where the traditional sexually transmitted diseases have not been controlled, the magnitude of problems associated with syphilis during pregnancy is reminiscent of that faced by the West during the early 1900 s.

(Sex Transm Inf 2000;76:73-79)

Keywords: syphilis; pregnancy

\section{Epidemiology}

Syphilis during pregnancy in the Western world is rare today. In this era, the prevalence of seropositivity in pregnancy is between 0.02 $4.5 \%$ in northern Europe and the United States after accounting for biological false reactive tests. ${ }^{1-6}$ Thanks to effective intervention strategies and the availability of penicillin, few of these pregnancies result in congenital syphilis-for example, an average of 30 cases per 100000 live births in the United States in 1996. ${ }^{7}$ The demographic profile of women who deliver syphilitic babies represents that of women with other sexually transmitted diseases (STDs) as well as those who fail to get adequate prenatal care. ${ }^{238}$ These women are more likely to be adolescent and unmarried. A disproportionate number of cases among childbearing women and their infants occur among minority groups - for example, African and Latin Americans in large urban areas. A major contributor to the increased occurrence is the use of crack cocaine ${ }^{2}$ and the exchange of sex for money with multiple partners. ${ }^{9}$

Incidence of syphilis increased dramatically in the former Iron Curtain countries during the past decade. ${ }^{10}$ For example, in Russia, the incidence of syphilis increased from $<30$ per 100000 in $1978-92$ to 175 per 100000 in 1995. The reason for this is unclear, but must be sought in sexual behaviour, increased travel, immigration, and prostitution, as well as provision, use, and effectiveness of diagnostic, treatment, and contact tracing services. Of particular concern is the decreased number of pregnant women screened for syphilis, partially due to reduced funding in some of these countries.

In parts of the world where the traditional "venereal diseases" have not been controlled, such as sub-Saharan Africa, the magnitude of the problems associated with congenital syphilis is reminiscent of those faced in the West during early $1900 \mathrm{~s}$. The prevalence of seroreactivity among pregnant women attending antenatal clinics in Africa is in the range of $3-18 \% .{ }^{11-15}$ There is uncertainty surrounding these figures. False reactive results occur because of cross reactivity with non-venereal treponemal infections. Non-venereal treponemal infections, such as yaws, reach a prevalence of $8 \%$ in some areas, such as Zaire. ${ }^{16}$ Scarce data on congenital syphilis in Africa suggested that $1-3 \%$ of the neonates and infants under 6 months of age are seroreactive and/or have signs of congenital infection. ${ }^{17}{ }^{18}$ In Ethiopia, an estimated $5 \%$ of all pregnancies are lost each year through syphilis induced abortions (75 000 pregnancy losses). ${ }^{19}$ In that same country, stillbirth among seroreactive women is five times more common than in seronegative women. In Zambia, 24\% of all stillbirths could be attributed to syphilis, and congenital syphilis was implicated in $30 \%$ of all perinatal mortality. ${ }^{20}$ In rural South Africa, an adverse pregnancy outcome in women with syphilis is 12 times more common than in seronegative women. ${ }^{15}$

\section{Clinical manifestations}

Syphilis is caused by the spirochaete Treponema pallidum. This organism is transmitted during sexual activity from a mucocutaneous lesion. 
The cervical changes, such hyperaemia, eversion, and friability, which occur during pregnancy may facilitate the entry and lead to spirochaetaemia. ${ }^{21}$ The fundamental histological changes of both congenital and acquired syphilis are vasculitis and its consequences, necrosis and fibrosis. Pregnancy has no known effect on the clinical course of syphilis. In acquired infection, after an initial incubation period of 3-90 days, a solitary papule with central ulceration, teeming with spirochaetes, erupts at the site of inoculation, which is often found on the genitalia, and less frequently on the rectal and the oral mucosa. This papular lesion is known as the chancre of syphilis and marks the primary stage of the disease. The chancre is accompanied by regional lymphadenopathy in $50 \%$ of the cases, and lasts from 4-6 weeks with spontaneous resolution. In about 2-6 weeks after the chancre resolves, systemic manifestations of the disease appear. These include headache, low grade fever, generalised lymphadenopathy, symmetrically distributed maculopapular rash found on the palms and the soles, the patchy motheaten alopecia seen with follicular scalp lesions, the highly infectious condyloma latum found on the genitalia, mild hepatitis, and nephrotic syndrome. The latent stage of the disease is characterised by reactive serological tests but no clinical manifestations. The latency is arbitrarily subdivided into early (1 year or less from the onset of the infection) and late (more than 1 year) latent stage. In the early latent stage, $25 \%$ of the patients will relapse with a secondary syphilitic manifestation whereas the chance for such relapses in the late latent stage is minimal. After years of untreated disease, one third of the adults can develop tertiary syphilis consisting of destructive lesions of the aorta (such as aortic aneurysm, regurgitation, luetic aortitis), central nervous system disorders (such as tabes dorsalis, meningiovascular syphilis, general paresis), skin and skeletal system manifestations (such as gummas).

The mother can transmit the infection transplacentally to the fetus or during passage through the birth canal by contact of the newborn with a genital lesion. Breast feeding does not result in the transmission of syphilis, unless an infectious lesion is present on the breast. Until recently, a commonly held but erroneous obstetric principle stated that infection of the fetus does not occur before 18 weeks. ${ }^{22}$ Silver and immunofluorescence staining of the fetal tissue, ${ }^{23}$ or polymerase chain reaction and rabbit infectivity testing of amniotic fluid ${ }^{24}$ showed that $T$ pallidum gains access to the fetal compartment as early as $9-10$ weeks. Untreated syphilis during pregnancy can profoundly affect pregnancy outcome, resulting in spontaneous abortion, stillbirth, non-immune hydrops fetalis, intrauterine growth restriction, premature delivery, and perinatal death, as well as serious sequelae in liveborn infected children. Most of the data on the outcome of pregnancy in untreated syphilis derived from observations in the era before penicillin was available or modern syphilis control programmes were operational in the Western world. These observations seem to simulate the present African conditions. In 1917, Osler observed that syphilis accounted for $20 \%$ of all stillbirths and $18-22 \%$ of the infant deaths in the United States. ${ }^{25}$ The same year, Harman published a report in England on the outcome 1001 pregnancies in 150 women with untreated syphilis and 826 pregnancies in a control group of 150 women with similar social status. ${ }^{26}$ Among the syphilitic group, $17.2 \%$ of the pregnancies resulted in a spontaneous abortion, $22.9 \%$ in newborn death, and $21 \%$ in congenital syphilis. Healthy infants were delivered in $38.9 \%$ of the cases, almost half of that in the control group. Two reports from the early 1950s confirmed these earlier observations and reveal the facts that the risk of prematurity, perinatal death, and congenital syphilis is directly related to the stage of maternal syphilis. ${ }^{27} 28$ In one study, ${ }^{27}$ among 22 women with untreated early syphilis of 4 years' duration or less, $41 \%$ of their infants had congenital syphilis, $25 \%$ were stillborn, $14 \%$ died in the neonatal period, and $21 \%$ were premature (defined as birth weight less that $5 \mathrm{lb}$ ), but had no evidence of congenital syphilis, and only $18 \%$ were well term infants. In this group, the possibility of neonatal death and stillbirth was, respectively, six and 32 times greater than that in the control group of 10323 pregnant uninfected women. Among 82 women with latent syphilis, only $2 \%$ of infants had congenital syphilis, $12 \%$ were stillborn, and $9 \%$ died in the neonatal period. In this group, only the stillbirth rate was greater than that in the uninfected control group, and $77 \%$ of the infants were full term and normal.

In the other study of 59 cases of untreated maternal syphilis, ${ }^{28}$ half of the mothers with primary or secondary syphilis delivered a premature or stillborn child, and the other half had syphilitic infants. The rate of congenital syphilis and perinatal accidents decreased slightly in early latent syphilis. With the late latent syphilis, approximately $10 \%$ of the infants were stillborn, and another $10 \%$ had congenital syphilis. There was no increase in the premature births or neonatal deaths beyond the expected rate of occurrence in women without syphilis.

Traditionally, congenital syphilis has arbitrarily been divided into two clinical syndromes: early and late congenital syphilis. Early congenital syphilis refers to those clinical manifestations that appear within 2 years of life. Those features that occur after 2 years, and usually manifest near puberty constitute late congenital syphilis. The clinical spectrum of congenital syphilis encompasses a wide spectrum of manifestations ranging from laboratory or radiographic abnormalities in the otherwise normal appearing newborn to severe disease involving multiple organs (table 1) A detailed discussion of manifestations occurring in untreated syphilitic children is beyond the scope of this review.

\section{Diagnosis}

In routine clinical practice, serological tests are usually used to make a diagnosis of gestational 
Table 1 Clinical presentations of congenital syphilis ${ }^{19}$

\begin{tabular}{ll}
\hline Clinical findings & Percentage \\
\hline Early & \\
Abnormal bone $x$ ray & 61 \\
Hepatomegaly & 51 \\
Splenomagaly & 49 \\
Petechiae & 41 \\
Skin leisons & 35 \\
Anaemia & 34 \\
Lymphadenopathy & 32 \\
Jaundice & 30 \\
Pseudoparalysis & 28 \\
Snuffles & 23 \\
Late & \\
Frontal bossing & $30-87$ \\
Palatal deformation & 76 \\
Dental dystrophies & 55 \\
Interstitial keratitis & $20-50$ \\
Abnormal bone $x$ ray & $30-46$ \\
Nasal deformity (saddle deformity) & \multirow{2}{*}{} \\
Eighth nerve deafness & $10-30$ \\
Neurosyphilis & $3-4$ \\
Joint disorder & $1-5$ \\
\end{tabular}

${ }^{\star}$ Hutchison triad.

syphilis, because most patients have no signs nor symptoms of disease. However, if a lesion such as chancre is present, dark field microscopy should be attempted to visualise the characteristic motile spirochaetes in the exudate collected from the lesion. This aids early diagnosis of the disease in some cases, because the appearance of a chancre precedes the conversion of serological tests by several days to 1 week. ${ }^{30}{ }^{31}$ Dark field microscopy has also been used to study amniotic fluid to confirm fetal infection. The findings of spirochaetes in the amniotic fluid may be a marker for more severe fetal disease. ${ }^{32}$ Specimens for dark field microscopy should be examined immediately after collection of the sample, since any delay in examination decreases the motility of spirochaetes, which is critical for the diagnosis. Immunofluorescent staining of $T$ pallidum is an alternative to dark field microscopy. ${ }^{33}$ Air dried exudate smeared on a microscope slide does not require immediate examination. Paraffin embedded biopsy or necropsy material can also be examined by immunofluorescent staining. ${ }^{34}$ Both dark field and immunofluorescence microscopy constitutes the most specific means of diagnosis provided that non-venereal treponemal infections such as yaws, bejel, and pinta are excluded. However, failure to find the organism does not exclude a diagnosis of syphilis. False negative results occur because of the age and condition of the lesion, ${ }^{35}$ treatment of the patients with antibiotics systemically or locally before the specimen collected and, most commonly, poor technique in collecting and reading the specimen. ${ }^{36}$

Serological tests for syphilis are classified into non-treponemal tests which include the venereal disease research laboratory (VDRL) and rapid plasma reagin (RPR) tests; and the treponemal tests, which include the fluorescent treponemal antibody absorption (FTA-ABS) assay and the microhaemagglutination assay for T pallidum antibody (MHA-TP). T pallidum immobilisation (TPI) assay, at one time the standard treponemal test in the United States, is now done in only a few research laboratories. Non-treponemal tests utilise an antigen comprising lecithin, cholesterol, and purified cardi- olipin (diphosphotidylglycerol), which is the constituent of both mammalian cell membranes and $T$ pallidum. ${ }^{37}$ The non-treponemal tests become reactive $4-8$ weeks after infection is acquired. ${ }^{30}{ }^{31}$ Their sensitivity for the diagnosis of primary and latent and late syphilis were in the range of $60-90 \% .^{38-40}$ With secondary syphilis, their sensitivity is close to $100 \%$. False negative results occur when testing the sera of less than $1 \%$ of the patients with primary or secondary syphilis. ${ }^{41}{ }^{42}$ This arises when there is an excess of treponemal antibodies in the tested sera resulting in the "prozone phenomenon". ${ }^{43}$ Diluting the serum before testing, thereby lowering the antibody concentration solves this problem. The rate of prozone phenomenon is very low, and routine serial dilutions are not cost effective. There are limited data suggesting that the prozone phenomenon may be more prevalent in HIV positive individuals with syphilis than in luetic patients who are negative for HIV. ${ }^{44}$ Thus serial dilution testing may be limited to those populations of patients with a high incidence of HIV positivity. Testing sera at cold temperaturesfor example, $4^{\circ} \mathrm{C}$, may also produce false negative reactions. Thus refrigerated sera must be warmed up-for example, to $27^{\circ} \mathrm{C}$, before testing. ${ }^{41}$

The non-treponemal tests react with sera from people who have never had treponemal infection. False positivity rate is around 1\% when screening the general population with these tests. ${ }^{38} 45-47$ False reactive results may be more frequent when testing certain patients groups, such as the elderly or the pregnant, or patients with drug addiction, malignancy, autoimmune diseases (for example, systemic lupus erythematosus), viral diseases (particularly with Epstein-Barr and hepatitis viruses), protozoal, or mycoplasma infection. ${ }^{31}{ }^{39}$ In low risk populations, all reactive test results should be confirmed by a treponemal test since over $50 \%$ of the non-treponemal tests may be false reactive.

Specific treponemal antibody tests include FTA-ABS and MHA-TP tests. The treponemal tests detect an antibody that reacts with antigenic compounds that are specific for the members of the genus Treponema. The FTAABS assay is highly sensitive (85-100\%) in all stages of the disease. ${ }^{36}{ }^{39} 46-48$ Compared with FTA-ABS assay, MHA-TP test is less sensitive $(60-85 \%)$ in the primary disease. ${ }^{3946} 48$ Studies where patients were simultaneously tested with treponemal and non-treponemal tests showed that the former are minimally more sensitive than the latter. ${ }^{48}{ }^{49}$ Since treponemal tests react despite successful treatment of syphilis, they should not be used in assessing serological response to therapy. False reactive results also occur with approximately $1 \%$ of uninfected individuals when screening the general population. ${ }^{45} 478$ False reactive test results can occur with connective tissue and autoimmune diseases, viral infections, pregnancy, ${ }^{39}$ and Lyme disease. ${ }^{29}$ There is also concern regarding occurrence of false negative results with serological tests for syphilis when testing immunosuppressed patients infected with HIV. 
Although seronegative results with specific treponemal tests have been documented in a few HIV infected cases with a history of syphilis in the past, ${ }^{50}{ }^{51}$ the serological response in HIV infected patients is often greater, possibly secondary to B cell dysregulation. ${ }^{44}$ Irrespective of the reports of false positive results of the non-treponemal and treponemal tests, expectant mothers should be treated if nontreponemal and treponemal tests are reactive and if a prompt and thorough evaluation of the cause of a possible false reactive result cannot be ensured.

New diagnostic tests include enzyme immunoassay (EIA), polymerase chain reaction (PCR), and immunoblotting. Of these, EIA based on the antibody capture method utilising (recombinant) treponemal antigen is commercially available. One such kit, the Captia Syph-G (Mercia Diagnostics, Guildford), which detects treponemal IgG, overall yielded a sensitivity of $100 \%$ and a specificity of $99 \%$ when testing pregnant women. ${ }^{52}{ }^{53}$ In primary syphilis, its sensitivity was $82 \%$, but this was nevertheless superior to those of treponemal and non-treponemal tests. ${ }^{54}$ Immunoblotting technique detecting specific treponemal IgG antibody, ${ }^{55} 56$ or polymerase chain reaction (PCR) detecting $T$ pallidum $\mathrm{DNA}^{24}{ }^{35}$ have proved to be highly sensitive and specific in experienced hands, but there are no standardised commercially available kits. Thus their use is currently confined to some research laboratories.

Prenatal laboratory diagnosis of fetal infection is possible. Since the maternal $\operatorname{Ig} M$ antibodies do not cross the placenta, detection of $\operatorname{IgM}$ antibodies in the fetal circulation is a consequence of fetal humoral response, and thus indicative of fetal infection. Wendel et al detected $\operatorname{Ig} M$ antibodies against $T$ pallidum using the immunoblotting technique on fetal blood collected by cordocentesis. ${ }^{57}$ PCR was used to detect $T$ pallidum DNA in the amniotic fluid collected early in the second trimester and was shown to be both sensitive and specific when compared with recovery of spirochaetes with rabbit infectivity test. ${ }^{35}$

Ultrasonography was used to detect some of the manifestations of syphilis in the fetus such as hydrops fetalis characterised by scalp oedema, placental thickening, serous cavity effusion, and polyhydramnios. ${ }^{58}$ Other ultrasonographic findings related to fetal syphilis include hepatosplenomegaly, placentomegaly, non-continuous gastrointestinal obstruction,

Table 2 Treatment guidelines for acquired syphilis during pregnancy ${ }^{62}$

\begin{tabular}{|c|c|}
\hline Stage of infection & Regimen \\
\hline $\begin{array}{l}\text { Primary } \\
\text { Secondary } \\
\text { Early latent ( }<1 \text { year) }\end{array}$ & Benzathine penicillin $G, 2.4$ million units intramuscularly $\times 1$ \\
\hline $\begin{array}{l}\text { Late latent ( }>1 \text { year) } \\
\text { Unknown duration }\end{array}$ & Benzathine penicillin G, 2.4 million units intramuscularly weekly $\times 3$ \\
\hline Neurosyphilis & $\begin{array}{l}\text { Aqueous penicillin } \mathrm{G}, 2-4 \text { million units intramuscularly every } 4 \text { hours } \\
\times 10-14 \text { days } \\
\text { or } \\
\text { Procaine penicillin } \mathrm{G} 2.4 \text { million units intramuscularly and probenecid } \\
500 \text { mg by mouth four times daily } \times 10-14 \text { days }\end{array}$ \\
\hline
\end{tabular}

and dilatation of the small bowel. ${ }^{59} 60$ Ultrasonographic signs of fetal syphilis indicate a greater risk for fetal treatment failure. Hepatomegaly, which is probably the initial sonographic manifestation of hydrops fetalis, seems to be the most sensitive ultrasonographic finding suggesting fetal infection. ${ }^{61}$

Doppler studies of both the uterine and umbilical arteries showed statistically significant increases in the mean systolic-diastolic ratios in syphilitic mothers compared with healthy controls. This indicates an increased resistance to perfusion of the placenta, probably secondary to focal areas of vasculitis and, similarly, placental villitis and obliterative arteritis in pregnancies complicated by syphilis. ${ }^{32}$

Routine screening of umbilical cord blood is not recommended. Serological testing of the mother's serum is preferred to testing infant serum, because the serological tests performed on infant serum can be non-reactive if the mother's serological test result is of low titre or if the mother was infected late in pregnancy. Testing umbilical cord blood might also yield a false positive result because it might be contaminated with maternal blood. No infant should leave the hospital without the maternal serological status having been documented at least once during pregnancy.

\section{Treatment}

Treatment during pregnancy should be with the penicillin regimen appropriate for the mother's stage of syphilis (table 2). Penicillin resistance has not been reported among any isolates. In a recent study involving 204 pregnant women with primary, secondary, or early latent syphilis, a single intramuscular dose of benzathin penicillin, 2.4 million units prevented fetal infection in $98 \%$ of the cases. ${ }^{63}$ In this cohort of patients, the only treatment failure of maternal infection occurred in a HIV positive woman. Nevertheless, some recommend a second dose of penicillin 1 week after the initial dose to ensure effective treatment. In one study involving 180 syphilitic women in South Africa, women receiving treponemicidal treatment for 3 weeks or less delivered infants with decreased birth weight, and had premature delivery, neonatal death, and syphilitic infants more frequently compared with those who were treated with a course of antibiotics for longer than 3 weeks. ${ }^{64}$ Although initial outcome was clearly improved at birth, complete cure rate could not be predicted from this study, because long term follow up of the neonates was not done.

Approximately $5-10 \%$ of pregnant women with syphilis report a history of penicillin allergy. ${ }^{65}$ Individuals at risk for acute allergic reactions can be identified by skin testing with major and minor determinants. If skin testing is positive, they can undergo intravenous or oral penicillin desensitisation. ${ }^{66}$ The oral route is preferred because it offers ease of administration and substantial cost savings. ${ }^{67}$ No serious reactions have been observed, and this method currently is recommended so that all pregnant women with penicillin allergy can receive 
penicillin treatment. After desensitisation patients must be maintained on penicillin continuously for the duration of the course of therapy.

There is no satisfactory alternative to penicillin for the treatment of syphilis during pregnancy. Erythromycin is not recommended because it frequently fails to eradicate syphilis in both the mother and the fetus. ${ }^{68-70}$ Tetracycline, the only other agent that has been proved effective, is not recommended because of dental staining and impairment of long bone growth in the fetus and hepatotoxicity when given intravenously (to a pregnant women with coexisting renal insufficiency). ${ }^{65}$ Insufficient data exist to recommend azithromycin or ceftriaxon for treatment of syphilis during pregnancy.

Monthly follow up of serological titres is recommended for the evaluation of the adequacy of the treatment and prevention of intrauterine infection. Following adequate therapy, nontreponemal tests in primary and secondary syphilis decline in titre fourfold after 3-6 months and eightfold after about 12 months. ${ }^{71}{ }^{72}$ With early latent infection, a fourfold decrease in titre occurs after 1 year. Patients treated for late latent or tertiary syphilis may have a more gradual decrease in titre. Low stable titres will persist in approximately $50 \%$ of these patients after 5 years. ${ }^{73}$ Pregnant women who were treated appropriately for syphilis in the past, but remain seroreactive should be treated again if sufficient evidence does not exist to ensure adequate treatment response - that is, Centers for Disease Control and Prevention define adequate response as (a) at least a fourfold decrease in non-treponemal antibody titres for patients treated for early syphilis, and (b) stable or declining nontreponemal antibody titres of less than or equal to $1: 4$ for other patients. ${ }^{62}$ Most women treated during pregnancy will deliver before their serological response to treatment can be assessed definitively. Neonates born to such women should be evaluated for congenital syphilis.

Pregnant women with reactive serological tests for syphilis should be counselled on the possibility of harbouring other sexually transmitted agents, and tested for these. Of particular importance is concomitant HIV infection. Data on concomitant syphilis and HIV infection are limited. Two prospective studies involving 178 non-pregnant syphilitic patients (95 HIV seropositive, 83 seronegative) found no clinical differences in clinical presentation, disease course, and response to therapy, but a lag in serological improvement in patients with HIV after therapy. ${ }^{74}$ However, a recent retrospective analysis found a potentially higher treatment failure among pregnant HIV positive patients. ${ }^{76}$ Thus some recommend a longer course of treatment such as 3 weeks for these patients.

Cerebrospinal fluid (CSF) abnormalities are common among HIV infected women patients who have primary or secondary syphilis, but these abnormalities are of unknown significance. ${ }^{77}$ Although of unknown benefit, some recommend CSF examination before and after therapy. CSF examination and retreatment should also be considered for patients in whom the suggested fourfold decrease in non-treponemal test titre does not occur within 3 months after treatment for primary or secondary syphilis as well as for all those with latent syphilis (regardless of apparent duration). In both circumstances a nonneurosyphilis regimen can be used if CSF examination is normal.

The Jarisch-Herxheimer reaction occurs in up to $45 \%$ of pregnant women after treatment for acquired early syphilis. It consists of fever, chills, myalgia, headache, hypotension, tachycardia, and transient accentuation of the cutaneous lesions. ${ }^{78}{ }^{79}$ It typically begins within several hours of treatment and resolves within 24-36 hours. The release of $T$ pallidum lipoprotein which possesses inflammatory activity from dead or dying organisms is implicated as a likely inducer of this phenomenon. In pregnant women, the Jarisch-Herxheimer reaction can cause uterine contractions and precipitate labour possibly mediated secondarily by prostaglandins. By fetal monitoring during the episode, evidence of fetal distress with fetal tachycardia and heart rate decelerations, along with a marked decrease in fetal activity, was demonstrated. No prophylactic measure of treatment is currently available. Thus some recommend hospitalisation of the mother during the first 24 hours after treatment. This allows early and timely intervention if fetal distress occurs. If fetal compromise is already present before treatment, and the fetus is viable, then delivery with subsequent treatment of the fetus and mother may result in improved outcome.

Despite administration of the recommended penicillin regimen to the pregnant women, as many as $14 \%$ will have a fetal death, or deliver infants with clinical evidence of congenital syphilis. ${ }^{768081}$ Many of these women have been treated for secondary syphilis late in pregnancy. Although these cases are recommended to be treated with at least two doses of benzathine penicillin 2.4 million units 1 week apart, the efficacy of this regimen in either preventing or fetal syphilis is not known. A severely infected fetus may be aborted in spite of the therapy. Mothers infected within 4 weeks of delivery may still deliver affected newborns. ${ }^{80} 82$ A possible explanation is that altered penicillin pharmacokinetics lead to lower serum and CSF levels of penicillin in both the mother and the fetus. ${ }^{83}$

\section{Prevention}

Public health strategies to prevent maternal and fetal syphilis are similar to those that focuses on syphilis and other STDs in the general population. These include early identification of infected individuals and high risk populations, adequate treatment, identification of the infected partners and their treatment, modification of high risk behaviour, and promoting the accessibility and the use of health care.

The leading factor accounting for the failure to prevent congenital infection is the lack of 
prenatal care. In the United States, $98.7 \%$ of pregnancies ending in a live birth have at least one prenatal medical visit ${ }^{84}$; in contrast, only $52 \%$ of mothers of infants with congenital syphilis reported having at least one prenatal visit. ${ }^{85}$ Among those mothers receiving prenatal care, the mean gestational age at which they were first seen for prenatal care was 22 weeks - that is, late in the second trimester. The likelihood of seeking prenatal care is strongly associated with age, marital and socioeconomic status, rural residence, and education attainment. Black mothers are twice as likely to receive delayed or no prenatal care. ${ }^{84}$ Community based programmes to reach out these groups and promoting their accessibility to prenatal care are likely to decrease the number affected with congenital syphilis.

Routine prenatal screening is the major line of defence against congenital syphilis. All pregnant women should have a non-treponemal serological test for syphilis during the first trimester. In areas with high indices of syphilis, serological screening should be performed at the beginning of the third trimester and at the delivery. ${ }^{86}$ When the high cost of long term institutional and medical care (annual average US cost was estimated to be $\$ 18.4$ million in $1995)^{87}$ as well as forgone economic output because of disability in severe congenital cases are considered, prenatal screening results in significant savings to society even when the prevalence of the maternal syphilis is as low as $0.005 .^{88-90}$ The addition of third trimester testing slightly increases the costs, but remains cost effective in high risk populations - for example, when the incidence of the maternal infection is greater than $0.02 .^{88}$ Premarital testing of couples was routinely performed in some countries after the second world war, and provided the only opportunity of identification of disease in those women who had had no prenatal tests, but has now been abandoned by and large, because it prevented very few additional cases at a very high cost - for example, an estimated $\$ 240000$ per case prevented in $1984 . .^{91}$

Identified cases should be counselled extensively about the disease, its impact on the pregnancy, and the importance of adequate treatment. Furthermore, they should be reported to the local public health department for contact investigation and appropriate follow up. Individuals who have had sexual contact with a woman with untreated syphilis or during the first 24 hours of treatment should be tested serologically, if possible, treated prophylactically, and retested 3 weeks later. Such management of partners is important to prevent not only the spread of the infection in the society, but also reinfection of the pregnant women.

1 Skarpaas T, Loe K. The occurrence of positive serologica syphilis reactions in pregnant women in Norway 1964 1978. Tidsskr Nor Laegeforen 1980;100:1840-3.

2 SisinCD, Ostrea EM, Reyes MP, et al. The resurgence of congenital syphilis; a cocaine-related problem. $\mathcal{F}$ Pediatr 1997;130:289-92.

3 Klass P, Brown E, Pelton S. The incidence of prenatal syphilis at the Boston City Hospital: a comparison across four decades. Pediatrics 1994;94:24-8.
4 Reyes M, Hunt N, Ostrea E, et al. Maternal/congenital syphilis in a large tertiary-care urban hospital. Clin Infect Dis 1993;17:1041-6.

5 Campos-Outcalt D, Ryan K. Prevalence of sexually transmitted diseases in Mexican-American pregnan women by country of birth and length of time in the United States. Sex Transm Dis 1995;22:78-82.

6 Bowell P, Mayne K, Puckett A, et al. Serological screening tests for syphilis in pregnancy: results of a five year study (1983-87) in the Oxford region. F Clin Pathol 1989;42. 1281-4.

7 Centers for Disease Control and Prevention. Sexually transmitted disease surveillance, 1996. Atlanta: Division of STD Prevention, US Department of Health and Human Services, Public Health Service, September 1997.

8 Mobley J, McKeown R, Jackson K, et al. Risk factors for congenital syphilis in infants of women with syphilis in South Carolina. Am f Public Health 1998;88:597-602.

9 Blank S, McDonnell D, Rubin S, et al. New approaches to syphilis control. Finding opportunities for syphilis treatment and congenital syphilis prevention in a women's correctional setting. Sex Transm Dis 1997;24:218-26.

10 World Health Organisation. HIV/AIDS and STD surveillance. Epidemiological fact sheets by country, 1998 . Geneva: WHO, 1998.

11 Azeze B, Fantahun M, Kidan K, et al. Seroprevalence of syphilis among pregnant women attending antenatal clinic-
sin a rural hospital in north west Ethiopia. Genitourin Med sin a rural hospital

12 Bam R, Cronje H, Muir A, et al. Syphilis in pregnant patients and their offspring. Int 7 Gynaecol Obstet 1994;44 $113-8$

13 Cossa H, Gloyd S, Vaz R, et al. Syphilis and HIV infection among displaced pregnant women in rural Mozambique. Int $\mathcal{F}$ STD AIDS 1994;5:117-23.

14 Lindstrand A, Bergström S, Bugalho A, et al. Prevalence of syphilis infection in Mozambican women with second trimestermiscarriage and women attending antenatal care in second trimester. Genitourin Med 1993;69:431-3.

15 Wilkinson D, Sach M, Connolly C. Epidemiology of syphilis in pregnancy in rural South Africa: opportunities forcontrol. Trop Med Int Health 1997;2:57-62.

16 Ziefer A, Lanoie L, Meyers W, et al. Studies on a focus of yaws in Ubangi, Zaire. Trop Med Parasitol 1985;36:63-71.

17 Hira S, Ratnam A, Bhat G, et al. Congenital syphilis in Lusaka-II. Incidence at birth and potential risks among hospital delivered infants. East Afr Med $\mathcal{F}$ 1982;59:306-8.

18 Hira S. Epidemiology of maternal and congenital syphilis in Lusaka and Copperbelt Provinces of Zambia. Lusaka, Zambia Republic of Zambia, 1984

19 Schulz K, Murphy F, Patamasucon P, et al. Congenital Syphilis. In: Holmes K, Mårdh P-A, Sparling P, et al, eds. Sexually transmitted diseases. New York: McGraw-Hill; 1990:821-42.

20 Schulz K, Cates W, O'Mara P. Pregnancy loss, infant death, and suffering: legacy of syphilis and gonorrhoea in Africa. Genitourin Med 1987;63:320-5.

21 Wendel G. Gestational and congenital syphilis. Clin Perinatol 1988;15:287-303.

22 Dippel A. The relationship of congenital syphilis to abortion and miscarriage, and the mechanism of uterine protection. Am $\mathcal{F}$ Obstet Gynecol 1944:369-79.

23 Harter C, Benirschke K. Fetal syphilis in the first trimester. Am $\mathcal{F}$ Obstet Gynecol 1976;124:705-11.

24 Nathan L, Bohman V, Sanchez P, et al. In utero infection with Treponema pallidum in early pregnancy. Prenat Diagn 1997;17:119-23.

25 Osler W. The anti-venereal campaign. Trans Med Soc Lond 1917;40:290.

26 Harman N. Staying the Plague. London: Methuen, 1917.

27 Ingraham N. The value of penicillin alone in the treatment of congenital syphilis. Acta Derm Venerol 1951;31:60-88.

28 Fiumara N, Fleming W, Dowing, et al. The incidence of prenatal syphilis at the Boston City Hospital. N Engl 7 Med 1952:247:48-52.

29 Carlsson B, Hanson H, Wasserman J, et al. Evaluation of the fluorescent treponemal antibody-absorption (FTA-Abs) test specificity. Acta Derm Venereol 1991;71:306-11.

30 Spangler A, Jackson J, Fiumara N. Syphilis with a negative blood test reaction. $\mathcal{f} A M A$ 1964;189:87-90.

31 Felman Y. How useful are the serologic tests for syphilis? Int f Dermatol 1982;21:79-81.

32 Lucas M, Theriot S, Wendel G. Doppler systolic-diastolic ratios in pregnancies complicated by syphilis. Obstet Gynecol 1991;77:217-22.

33 Cummings M, Lukehart S, Marra C, et al. Comparison of methods for the detection of Treponema pallidum in methods for the detection of Treponema palidum

34 Rawstron S, Vetrano J, Tannis G, et al. Congenital syphilis: detection of Treponema pallidum in stillborns. Clin Infect Dis 1997;24:24-7.

35 Zoechling N, Schluepen E, Soyer H, et al. Molecular detection of Treponema pallidum in secondary and tertiary syphilis. Br f Dermatol 1997;136:683-6.

36 Romanowski B, Forsey E, Prasad E, et al. Detection of Treponema pallidum by a fluorescent monoclonal antibody test. Sex Transm Dis 1987;14:156-9.

37 Belisle J, Brandt M, Radolf J, et al. Fatty acids of Treponema pallidum and Borrelia burgdorferi lipoproteins. $\mathcal{F}$ Bacteriol 1994;176:2151-7.

38 Phaosavasdi S, Snidvongs W, Thasanapradit P, et al. Rapid plasma reagin test (RPR) compared to venereal diseases research laboratory test (VDRL) for the diagnosis of syphilis in pregnancy. $\mathcal{F}$ Med Assoc Thai 1989;72:202-6. 
39 Jaffe $\mathrm{H}$, Musheer D. Management of the reactive syphilis serology. In: Holmes $\mathrm{K}$, Mårdh $\mathrm{P}-\mathrm{A}$, Sparling $\mathrm{P}$, et al, eds. Sexually transmitted diseases. New York: McGraw-Hill, 1990:935-9.

40 Hambie E, Larsen S, Perryman M, et al. Comparison of a new rapid plasma reagin card test with the standard rapid plasma reagin $18-\mathrm{mm}$ circle card test and the venereal disease research laboratory slide test for serodiagnosis of syphilis. f Clin Microbiol 1983;17:249-54.

41 el-Zaatari M, Martens M. False-negative syphilis screening due to

42 el-Zaatari $M$, Martens $M$, Anderson G. Incidence of the prozone phenomenon in syphilis serology. Obstet Gynecol prozone phenom

43 Berkowitz K, Baxi L, Fox H. False-negative syphilis screening: the prozone phenomenon, nonimmune hydrops, and diagnosis of syphilis during pregnancy. Am $\mathscr{f}$ Obstet Gynecol 1990;193:975-7.

44 Jurado R, Campbell J, Martin P. Prozone phenomenon in secondary syphilis. Has its time arrived? Arch Intern Med 1993;153:2496-8.

45 Goldman J, Lantz M. FTA-ABS and VDRL slide test reactivity in a population of nuns. $\mathcal{f} A M A 1971 ; 217: 53-5$.

46 Jaffe $\mathrm{H}$, Larsen S, Jones $\mathrm{O}$, et al. Hemagglutination tests for syphilis antibody. Am f Clin Pathol 1978;70:230-3.

47 Wentworth B, Thompson M, Peter C, et al. Comparison of a hemagglutination treponemal test for syphilis (HATTS) with other serologic methods for the diagnosis of syphilis. Sex Transm Dis 1978;5:103-14.

48 Huber T, Storms S, Young P, et al. Reactivity of microhemagglutination, fluorescent treponemal antibody plasma reagin tests in primary syphilis. 7 Clin Microbio 1983;17:405-9.

49 Dyckman J, Storms S, Huber T. Reactivity of microhemagglutination, fluorescent treponemal antibody absorption, and venereal disease research laboratory tests in primary syphilis. F Clin Microbiol 1980;12:629-30.

50 Johnson P, Graves S, Stewart L, et al. Specific syphilis serological tests may become negative in HIV infection. AIDS 1991;5:419-23.

51 Erbelding E, Vlahov D, Nelson K, et al. Syphilis serology in human immunodeficiency virus infection: evidence for false-negative fluorescent treponemal testing. F Infect Dis false-negative fluoresce

52 Silletti R. Comparison of CAPTIA syphilis G enzyme immunoassay with rapid plasma reagin test for detection of immunoassay with rapid plasma reagin test

53 Ross J, Moyes A, Young H, et al. An analysis of false positive reactions occurring with the Captia Syph G EIA Genitourin Med 1991:67:408-10.

54 Lefevre J, Bertrand M, Bauriaud R. Evaluation of the Captia enzyme immunoassays for detection of immunoglobulins $\mathrm{G}$ and $\mathrm{M}$ to Treponema pallidum in syphilis. $\mathcal{F}$ Clin Microbiol 1990;28:1704-7.

55 Dettori G, Grillo R, Mora G, et al. Evaluation of western immunoblotting technique in the serological diagnosis of human syphilitic infections. Eur F Epidemiol 1989·5·22-30.

56 Byrne R, Laska S, Bell M, et al. Evaluation of a Treponema pallidum western immunoblot assay as a confirmatory test for syphilis. f Clin Microbiol 1992;30:115-22.

57 Wendel G, Sanchez P, Peters M, et al. Identification of Treponema pallidum in amniotic fluid and fetal blood from
pregnancies complicated by congenital syphilis. Obstet pregnancies complicated

58 Levine Z, Sherer D, Jacobs A, et al. Nonimmune hydrops fetalis due to congenital syphilis associated with negative intrapartum maternal serology screening. $A m \mathcal{F}$ Perinato 1998;15:233-6.

59 Hill LM JB. An unusual constellation of sonographic findings associated with congenital syphilis. Obstet Gynecol 1991;78:895-7.

60 Satin A, Twickler D, Wendel G. Congenital syphilis associated with dilation of fetal small bowel. A case report. $\mathcal{F}$ Ultrasound Med 1992;11:49-52.

61 Nathan L, Twickler D, Peters M, et al. Fetal syphilis: correlation of sonographic findings and rabbit infectivity testing of amniotic fluid. F Ultrasound Med 1993;12:97-101.

62 Centers for Disease Control and Prevention. 1998 Guidelines for treatment of sexually transmitted diseases. lines for treatment of sex

63 Alexander J, Sheffield J, Sanchez P, et al. Efficacy of treatment for syphilis. Obstet Gynecol 1999;93:5-8.
64 Donders G, Desmyter J, Hooft P, et al. Apparent failure of one injection of benzathine penicillin $\mathrm{G}$ for syphilis during pregnancy in human immunodeficiency virus-seronegative African women. Sex Transm Dis 1997;24:94-101.

65 Sanchez P, Wendel G. Syphilis in pregnancy. Clin Perinat 1997;24:71-90.

66 Wendel G, Stark B, Jamison R, et al. Penicillin allergy and desensitization in serious infections during pregnancy. $N$ Engl f Med 1985;312:1229-32.

67 Chisholm C, Katz V, McDonald T, et al. Penicillin desensitization in the treatment of syphilis during pregnancy. $A m \mathcal{F}$ Perinatol 1997;14:553-4.

68 Fenton L, Light I. Congenital syphilis after maternal treatment with erythromycin. Obstet Gynecol 1976;47:4924.

69 Hashisaki P, Wertzberger G, Conrad G, et al. Erythromycin failure in the treatment of syphilis in a pregnant woman. Sex Transm Dis 1983;10:36-8.

70 Duncan W. Failure of erythromycin to cure secondary syphilis in a patient infected with the human immunodeficiency virus. Arch Dermatol 1989;125:82-4.

71 Fiumara N. Standards for treatment of primary and secondary syphilis. Am Fam Physician 1983;27:185-8.

72 Hart G. Syphilis tests in diagnostic and therapeutic decision making. Ann Intern Med 1986;104:368-76.

73 Fiumara N. Serologic responses to treatment of 128 patient with late latent syphilis. Sex Transm Dis 1979;6:243-6.

74 Gourevitch M, Selwyn P, Davenny K, et al. Effects of HIV infection on the serologic manifestations and response to treatment of syphilis in intravenous drug users. Ann Intern Med 1993;118:350-5.

75 Yinnon A, Coury-Doniger P, Polito R, et al. Serologic response to treatment of syphilis in patients with HIV infection. Arch Intern Med 1996;156:321-5.

$76 \mathrm{McFarlin} \mathrm{B}$, Bottoms S, Dock B, et al. Epidemic syphilis: maternal factors associated with congenital infection. $A m \mathcal{F}$ Obstet Gynecol 1994;170:535-40.

77 Tomberlin M, Holtom P, Owens J, et al. Evaluation of neurosyphilis in human immunodeficiency virus-infected individuals. Clin Infect Dis 1994:18:288-94.

78 Klein V, Cox S, Mitchell M, et al. The Jarisch-Herxheimer reaction complicating syphilotherapy in pregnancy. Obste Gynecol 1991;75:375-80.

79 Myles T, Elam G, Park-Hwang E, et al. The JarischHerxheimer reaction and fetal monitoring changes in pregnant women treated for syphilis. Obstet Gynecol 1998;92 859-64.

80 Mascola L, Pelosi R, Alexander C. Inadequate treatment of syphilis in pregnancy. Am f Obstet Gynecol 1984;150:945-7.

81 Conover C, Rend C, Miller G, et al. Congenital syphilis after treatment of maternal syphilis with a penicillin regimen 6:134-7.

82 Chhabra R, Brion L, Castro M, et al. Comparison of maternal sera, cord blood, and neonatal sera for detecting presumptive congenital syphilis: relationship with materna treatment. Pediatrics 1993;91:88-91.

83 Nathan L, Bawdon R, Sidawi J, et al. Penicillin levels following the administration of benzathine penicillin $G$ in pregnancy. Obstet Gynecol 1993;82:338-42.

84 National Center for Health Statistics, US Department of Health and Human Services. Health aspects of pregnancy and childbirth: United States, 1980-1985. Vital and Health Statistics 1995; Series 23: No 18.

85 Centers for Disease Control and Prevention. Division of STD Prevention. Current Trends. Congenital syphilisSTD Prevention. Current Trends. Congenital syphil

86 Opai-Tetteh E, Hoosen A, Moodley J. Re-screening for syphilis at the time of delivery in areas of high prevalence. $S$ Afr Med F 1993;83:725-6.

87 Bateman D, Phibbs C, Joyce T, et al. The hospital cost of congenital syphilis. F Pediatr 1997;130:752-8.

88 Stray-Pedersen B. Economic evaluation of maternal screening to prevent congenital syphilis. Sex Transm Dis 1983;10: $167-72$.

89 Williams K. Screening for syphilis in pregnancy: an assessment of the costs and benefits. Commun Med 1985;7: $37-42$.

90 Abyad A. Cost-effectiveness of antenatal screening for syphilis. Health Care Women Int 1995;16:323-8.

91 Haskell R. A cost-benefit analysis of California's mandatory premarital screening program for syphilis. West $\mathcal{f}$ Med 1984;141:538-41. 